\title{
Constraints from Carbon Cycle Perturbations on the Sensitivity of Runoff to Atmospheric $\mathrm{CO}_{2}$
}

\author{
JEREMY K. CAVES RUGENSTEIN ${ }^{1,2,3}$ AND ALEXANDER \\ WINKLER $^{1}$
}

${ }^{1}$ Max Planck Institute for Meteorology, Hamburg, Germany (jeremy.rugenstein@mpimet.mpg.de; alexander.winkler@mpimet.mpg.de)

${ }^{2}$ Senckenberg Biodiversity and Climate Research Center, Frankfurt, Germany

${ }^{3}$ Department of Geosciences, Colorado State University, Fort Collins, CO, USA (jeremy.rugenstein@ colostate.edu)

Earth history is punctuated by abrupt climatic perturbations that have frequently been caused by severe, rapid perturbations to the surface Earth carbon cycle, such as from eruptions of Large Igneous Provinces or rapid release of methane hydrates, among other possible mechanisms. However, despite the potentially varied mechanisms that may cause carbon cycle perturbations, the Earth system has seemingly always recovered from such perturbations relatively rapidly. Such recovery is frequently attributed to an increase in hydrological cycling that subsequently increases the silicate weathering flux, thereby removing excess carbon-the so-called silicate weathering negative feedback. We use the recovery from such carbon cycle perturbations as well as an improved mechanistic understanding of silicate weathering to place constraints on the hydrological response to increasing atmospheric $\mathrm{CO}_{2}$. To do so, we embed recently developed mechanistic equations that link climate, runoff, and weathering into a long-term carbon cycle model to constrain the sensitivity of global runoff to atmospheric $\mathrm{CO}_{2}$. We find that this sensitivity must always be positive, such that globally-averaged runoff increases in response to an increase in atmospheric $\mathrm{CO}_{2}$. Such positive sensitivity holds several surprising implications: (1) though runoff comprises the small residual difference between precipitation and evapotranspiration on land, the positive change in precipitation will always be greater than the positive change in evapotranspiration, and; (2) tectonic configuration matters tremendously in determining the runoff response, and thereby matters equally to land surface reactivity in determining how quickly the carbon cycle recovers from a perturbation. Lastly, we calculate the runoff sensitivity required to match the $\delta^{13} \mathrm{C}$ recovery from the PETM, and find that the required runoff sensitivity is substantially higher than that predicted by the latest generation of global climate models participating in CMIP. 